\title{
Commentary on "The role of conventional pelvic floor reconstructive surgeries in obstructed defecation symptoms change: CARE and OPTIMAL trials sub-analysis of 2-year follow-up data"
}

\author{
Abdelmageed Abdelrahman ${ }^{1}$ \\ Received: 24 January 2020 / Accepted: 27 January 2020 / Published online: 15 February 2020 \\ (C) The International Urogynecological Association 2020
}

This study [1] was aimed at addressing two main questions: is any conventional vaginal prolapse repair effective in curing obstructed defecation symptoms and is there evidence to suggest that a sacrocolpopexy will increase the risk of worsening or newly onset obstructed defecation symptoms? The authors used a sub-analysis of two major clinical trials performed by the Pelvic Floor Disorders Network: the Colpopexy and Urinary Reduction Efforts (CARE) trial and the Operations and Pelvic Muscle Training in the Management of Apical Support Loss (OPTIMAL) trial. The study included all patients from both trials who answered the PFDI-20 questionnaire and had undergone a POP-Q examination at baseline and at 24 months.

In total, 353 subjects from the OPTIMAL study and 279 subjects from CARE study met the inclusion criteria. Obstructed defecation symptoms were present in more than half of the patients at the initial visit before surgical intervention and approximately one third of patients were symptomatic at the 24-month follow-up in all surgical groups.

The authors concluded that conventional vaginal prolapse surgeries, with or without posterior vaginal wall repair (including uterosacral ligament suspension, sacrospinous ligament suspension and abdominal sacrocolpopexy), improved obstructed defecation symptoms by 50\%; however, approximately $35 \%$ of patients were suffering from at least one of the aspects of obstructed defecation at 24 months. The authors also highlighted that approximately one quarter of patients experienced persisting or worsening of their obstructed defecation symptoms in the absence of anatomical failure.

The authors present an interesting discussion, but the study has some weaknesses. Importantly, they analyse two major clinical trials whose primary purpose was not to study obstructive defecatory symptoms. Additionally, subjects in both clinical trials did not undergo the complete pre-operative evaluation of obstructive defecatory symptoms and ultrasound assessment of their anatomy.

\section{Compliance with ethical standards}

Conflicts of interest None.

\section{Reference}

1. Rostaminia G, Abramowitch S, Chang C, Goldberg RP. The role of conventional pelvic floor reconstructive surgeries in obstructed defecation symptoms change: CARE and OPTIMAL trials sub-analysis of 2-year follow-up data. Int Urogynecol J. 2019; https://doi.org/10. 1007/s00192-019-04190-7

Publisher's note Springer Nature remains neutral with regard to jurisdictional claims in published maps and institutional affiliations.

Abdelmageed Abdelrahman

abdelmageed@hotmail.co.uk

1 Department of Urogynaecology, Liverpool Women's Hospital NHS Foundation Trust, Liverpool, UK 